\title{
An Exploration of the Application of Communication Technology and Network in Computer Control
}

\author{
Deji Shang
}

Open Education Institute of Zhongzhou University

\begin{abstract}
With the constant development of the economy, the high-speed development of network technique and computer communication technology as well as the continuous development of communication technology and network technique keep renewing computer automatic system, which has been applied to many fields, providing the basis and guarantee for the improvement and development of the society. The thesis mainly analyzes the communication technology and network technique in computer control, and thus boosts the development of network technique and communication technology and ensures the quality of life and services.
\end{abstract}

Key words- Application of communication technology and network in computer control

\section{INTRODUCTION}

With the continuous development of science and technology, computer information technology becomes widespread in people's daily life, which is very important for the development of society and can boost the improvement of daily life. Seen from the history of computer development in China, the high-speed development stage is in 1990s. Based on the constant and high-speed development of digital technique, digital communication technology and switching technology, traditional analog signals are gradually transformed into digital signals, which then form digital communication technology. With the continuous development of the society, there have been some achievements in micro-electronics and computer technology, which will accelerate the fusion of information communication technology and computer networking technology.

\section{Basic summary of communication technology and computer network}

(I) Network technique

In fact, network technique means to combine communication technology and computer technology organically and connect computer lines and communication devices reasonably within different zones, thus achieving the purposes of resource transmission and resource sharing. Network technique is mainly composed of two parts: network resource and communication device. Generally speaking network is a computer which combines connecting telephone wire, wireless communication and cable etc. Expedite communication is able among network nodes and thus the software, hardware and the database in the shared network can play their roles. In general complete computer network is 
mainly composed of repeater, network bridge, Hub, Router, Switch and gateway etc.

\section{(II) Computer communication technology}

Generally speaking computer communication means the basic processes which connects the transmission and interactive information between computers and between computer and devices. Seen from the historical development of communication technology, the development process mainly contains analog communication era, digital communication era and data communication era. Analog signal is the carrier of analog communication transmission; digital signal is the carrier in the digital communication era and active information data is the carrier in data communication era. Based on such communication technology can be divided into three switching modes including packet switching, circuit switching and message switching. The major equipment for information sharing between transmission data and computer in network is networking protocol. Actually networking protocol is a language acknowledged by both. For different computers, the reasonable application of identical networking protocol is important for the transmission and sharing of data information. Choose networking protocols reasonably according to the practical situation and the networking protocol is changeable to some extent [1].

\section{Types of applied network technique and communication technology in computer control}

\section{(I) Field bus technology}

Field bus technology possesses relatively scattered function modules, which can effectively enhance system reliability and provide the basis and guarantee for further system maintenance. The chief application of field bus technology is open interconnection architecture and can interconnect computer networks at the same level. Through practice it can be found that field bus technology has proven technique, stronger security, stability and compatibility etc. However, upon practical application, due to the influence and restriction of its own conditions, there are some shortcomings and defects like low efficiency, low-speed resource sharing and transmission data, difficulties in connecting computer internet and relatively complex bus implementation standard, which often lead to control difficulties.

\section{(II) Ethernet}

Ethernet is the most extensively applied technological mode in computer control technology. Compared with field bus technology it is different and its transmission speed and transmission efficiency are much higher. Ethernet also has strong compatibility; it will not be subject to the restrictions of external factors and its transmission process will not be affected. However, in actual application, its reliability and security are worse than those of field bus technology; as a result, Ethernet cannot have effective control over field devices. So it is necessary to combine both technologies mentioned above organically in the actual application of network technique in order to give full play to the advantages of both technologies, avoid their disadvantages and guarantee the maximization of the efficiency of system devices. In addition, the application of network communication technology can use energy effectively and lower resource consumption to some extent, thus helping to achieve the purpose of information and resource sharing. 
From teaching aspect, the use of network communication technology can enhance the efficiency of distance teaching and guarantee effective transmission and the exchange culture. Distance teaching based on computer networking technology will not only break traditional teaching mode and help to establish new space for domestic educational cause, but also be able to expand the coverage of cultural education constantly, ensure effective communication of various ethnic groups' cultures and more fixed fusion of domestic cultures and do good to common prosperity and development. The constant development of network technique and communication technology can improve the communication and exchange among different countries and boost their continuous progress and development. Besides, because there are many ethic groups in China and different ethnic groups have their own life and religious beliefs, the application of network communication technology will help to protect and inherit their cultures in the future and prevent them from being damaged in the trend of diversified world development. From the aspect of meteorological observation, communication network technique has a certain role in actual application. By means of satellite, broadcast and radio station etc, it can transmit meteorological changes timely and effectively, and thus people are able to have a good understanding of weather conditions and the protection work can be guaranteed and put into practice [2].

\section{Application of communication technology and network technique in computer control}

With the rapid development of communication technology and network technique, they are applied to all walks of life more frequently. The role communication technology and network technique play in computer control can be analyzed from the following aspects [3]. First, they can lower resource consumption greatly and ensure more effective data and information sharing. People can obtain and store the data information, images and voice in computer network more conveniently with the terminal equipment, thus realizing the purpose of enhance transmission efficiency effectively, avoiding frequent and repeated data input, lowering the financial, material and manual labor consumption in arising in data storage and ensuring the value of network information resources can be played fully. Second, use network communication technology more reasonably in distance teaching to achieve the purpose of exchanging and disseminate culture. For instance, communication satellite should be used reasonably in distance teaching in order to guarantee a wide educational outreach; distance teaching is an effective supplementary means to traditional teaching methods and is of significance for vocational education, adult education and life-long education. Moreover, it will accelerate the development of open teaching modes, enlarge the range of education and help to cultivate more talents. Third, in meteorological aspect, communication technology and network technique are of certain significance; they can transmit the actual meteorological changes. Based on this, in the process of social development, communication technology and network technique occupy an important position, influence all aspects of society and have a certain development prospect [4].

\section{Development of network and communication technology in China}

In recent years, with the rapid development of 
$\begin{array}{llr}\text { communication } & \text { technology and network } \\ \text { technique, } & \text { nationwide } & \text { integrated }\end{array}$ communication network has covered communication network basically. It needs to improve and optimize corresponding network devices and facilities in order to guarantee a leading network size in the world. Firstly, communication technology and network technique are restricted and influenced by computer control technology. In the 1980s, the first analog paging system was established in Shanghai and the first digital paging system of China was set in Guangzhou. The development and progress of information technology and network technique have a close relationship with computer control technology. In addition, computer control technology stimulates the popularization and development of integrated circuit as well as the development of mobile terminal devices including transmitter and receiver etc. The constant reformation and improvement of communication technology and network technique provide the foundation and guarantee for the development and design of high-capacity SPC exchange. Maintenance and control information technologies change the pre-control pattern of traditional control equipment and keep optimizing the sequencing and automation of control. Moreover, it is also able to store content in the storage area. After exchange is completed, the number used by control must be changed and added constantly based on this; implement corresponding programs in accordance with the practical situation and design requirements and ensure various functions can be exchanged effectively. The SPC exchange can be under effective control based on computer control technology. Secondly, the constant reformation of communication technology accelerates the progress and development of communication technology and network technique. Communication technology and network technique are widely applied in China, and people keep accepting new communication modes. During the process of communication mode change, more diversified network technique and communication applications appear in computer control technology, and central system, distributed system and embedded system are increasingly used in computer control [5].

\section{Conclusion}

In conclusion, with the deepened development of computer technology and internet, people's daily life is influenced and changed to a great extent; people gradually step toward an intelligent, efficient and simplified society. The application of computer communication technology in daily life and production can ensure that people's life becomes more convenient. For example, the development of mobile phone television makes communication between people freer; the development of video call is able to enrich people's daily life; the development of digital television can enlarge the coverage. Seen from the actual development of the society, internet will be essential in future development and it can change the way of society informatization to some extent; the constant application of communication technology and network technique in real life and production and the formation of artificial intelligence mode will change people's lifestyle to a great extent; the gradual development of technologies including video call and mobile internet toward IP direction will bring more business types and patterns, providing basis and guarantee for the fusion and development of computer control technology and communication network technique. From the 
angle of social development, the constant improvement of the strength and speed of information networks does good to the development and progress of social harmony, fusion and unification.

\section{Reference}

[1] Dong Qiao, Network and communication technology in computer control [J], Digital Technology and Application, vol. 12, pp. 42-42, 2011.

[2] Jing $\mathrm{Wu}$, Yudan Dong, Application of network and communication technology in computer control [J], Electronic Test, vol. 14, pp. 101-103, 2014.
[3] Xinwei Meng, Xiang Zhao, An exploration and analysis of network and communication technology in computer control [J], Science \& Technology and Enterprise, vol. 15, pp. 102-102, 2013.

[4] Hai Li, An exploration of network and communication technology in computer control [J], China New Telecommunications, vol. 21, pp. 42-42, 2014.

[5] Yuehua Huang, Hongbing Cheng, On the network and communication technology in computer control [J], Technology Innovation and Application, vol. 32, pp. 70-70, 2013. 